\title{
Can the Oxidative Stress Index Predict the Severity of COVID-19?
}

\author{
Harold I. Zeliger and Harvey Kahaner
}

\begin{abstract}
Severity of the COVID-19 disease ranges from imperceptible to death with the aged and those with preexisting conditions being particularly vulnerable to severe symptoms. Other factors have also been shown to influence COVID-19 severity. These include smoking, vaping and exposure to air pollution. These factors have a one thing in common, all raise oxidative stress. The Oxidative Stress Index, derived from a questionnaire and reflective of oxidative stress level, is proposed as a non-invasive way to predict the severity
\end{abstract} of COVID-19 in those impacted by the Coronavirus.

Index Terms - Corona virus; COVID-19; COVID-19 severity; Oxidative stress; Oxidative stress index.

\section{INTRODUCTION}

Oxidative stress (OS), a state of imbalance between free radicals and antioxidants in the body, is well known as a direct cause of non-communicative (environmental) disease as well as an indirect cause of bacterial and viral-causing disease via undermining of the immune system (Zeliger, 2016; Rajah \& Chow, 2015; Zaki, et al., 2005). The Oxidative Stress Index (OSI), a non-invasive, questionnairebased index which numerically quantifies OS, has been shown to be an indicator of the environmental disease severity (Zeliger, 2017). It is proposed here that the OSI can be used to predict disease severity of communicative diseases such as COVID-19.

As originally designed the OSI is a $400+$ question entity that incorporates all causes of environmental diseases. These include; age, gender, body weight, genetics and epigenetics, prevalent diseases, symptoms regularly experienced, pharmaceuticals used, toxic chemical exposure, radiation exposure, chronic inflammation and lifestyle choices (including, diet, smoking, regular alcohol consumption, recreational drug use and psychological stress).

Recently, the OSI has been reduced to a condensed, eightparameter questionnaire that can be rapidly completed by a patient or surrogate to yield results that are within ninety percent of those obtained from the original long form questionnaire (Zeliger 2020). As such, the condensed OSI lends itself to use as a survey tool to predict disease severity.

The OSI may also serve to explain why severe symptoms of COVID-19 are being observed in some people aged 2044 who, based on the presence of pre-existing conditions and age, are expected to be asymptomatic or have only mild symptoms.

Published on April 16, 2020.

H. I. Zeliger (Ph. D., Corresponding Author) and Harvey Kahaner (M. D.) are with Zeliger Research and Consulting, 41 Wildwood Drive, Cape Elizabeth, Maine 04107, USA (e-mail: hiz@zeliger.com)

\section{METHODS}

The hypothesis presented here is based upon a literature review of numerous publications on the association between OS and disease, as well as the use of the OSI as a predictor of the likelihood of disease onset and severity.

\section{RESUltS AND DisCUSSION}

\section{A. Oxidative Stress}

Oxidative stress is a state of imbalance of free radicals and antioxidants in the body. The numerous causes of OS include age, gender, body weight, genetics and epigenetics, prevalent diseases, disease symptoms regularly experienced, pharmaceuticals used, toxic chemical exposure, radiation exposure, chronic inflammation and lifestyle choices (including, diet, smoking, alcohol and recreational drug use) and psychological stress (Zeliger, 2016 and the numerous references contained therein).

Chronic OS is associated with the onset of numerous noncommunicative diseases, including type 2 diabetes, heart diseases, respiratory diseases, neuro-degenerative diseases, neuro-developmental diseases, immunological disorders and skeletal disorders (Zeliger, 2016).

OS is also associated with the onset and severity of infectious viral, bacterial, fungal and parasite-carried diseases via immune system suppression (Hughes, 1999; Akaike, 2001; Splettstoesser \& Schuff-Warner, 2002; Xu, et al., 2015).

It is well established that the onset of chronic disease leads to multi-morbidity. People with non-communicative diseases subsequently have a high incidence of other noncommunicative diseases, as well as more frequent and severe bouts with infectious diseases (Zeliger, et al., 2012; Zeliger, 2014; Andresen, et al., 2006). Viral infections have been shown to be co-morbid with Alzheimer's disease (Honjo, et al., 2009). Co-morbidity has also been established between tuberculosis or malaria and type 2 diabetes (Marais, et al., 2013).

OS is associated with lipid peroxidation, protein and DNA damage (Pizzino, et al., 2017). Lipid peroxidation biomarkers that are typically used to measure OS include F2-iso-prostanes, lipid hydroxides and hydroperoxides, aldehydes and ketones. Of these, malondialdehyde (MDA) level in serum is the most commonly used biomarker to determine OS levels. MDA is stable in serum and is readily and accurately analyzed. Elevated MDA levels are associated with all OS raising parameters.

MDA, as well as other biomarkers are point in time measurements only and can vary up and down as much as $19 \%$ over a six-day period, depending upon factors such as 
diet, exposure to ambient air pollutants, psychological stress and other life-style factors (Nielsen at al., 1997). In addition, MDA measurements require time consuming laboratory analysis that is not universally available. The Oxidative Stress Index (OSI) was developed to overcome these shortcomings.

\section{B. Oxidative Stress Index (OSI)}

The OSI is based upon a yes/no questionnaire that a patient or surrogate can complete. The OSI as originally developed contains $400+$ items that address all causes of OS enumerated above. The number of positive items checked add up to the OSI and the higher the OSI, the greater the likelihood of disease onset (Zeliger, 2017). Since its original publication, the OSI has been applied to predicting causes of specific disease, carrying out public health surveys to asses the impact of proximate chemical discharges, health prescreening and limited medical care area screening (Zeliger 2019).

\section{Condensed OSI Form}

The OSI in its original format, can be used to show which specific OS raising parameters need to be addressed to reduce the likelihood of disease onset. At $400+$ items, however, considerable time is required for its completion and it has been found to be cumbersome for regular use in clinical settings. To overcome this shortcoming, a new eight item version of the OSI that addresses the causes of OS elevation has been developed (Zeliger, 2020). This condensed version is shown in Table I.

TABLE I: OXIDATIVE STRESS INDEX (OSI) CONDENSED FORM*

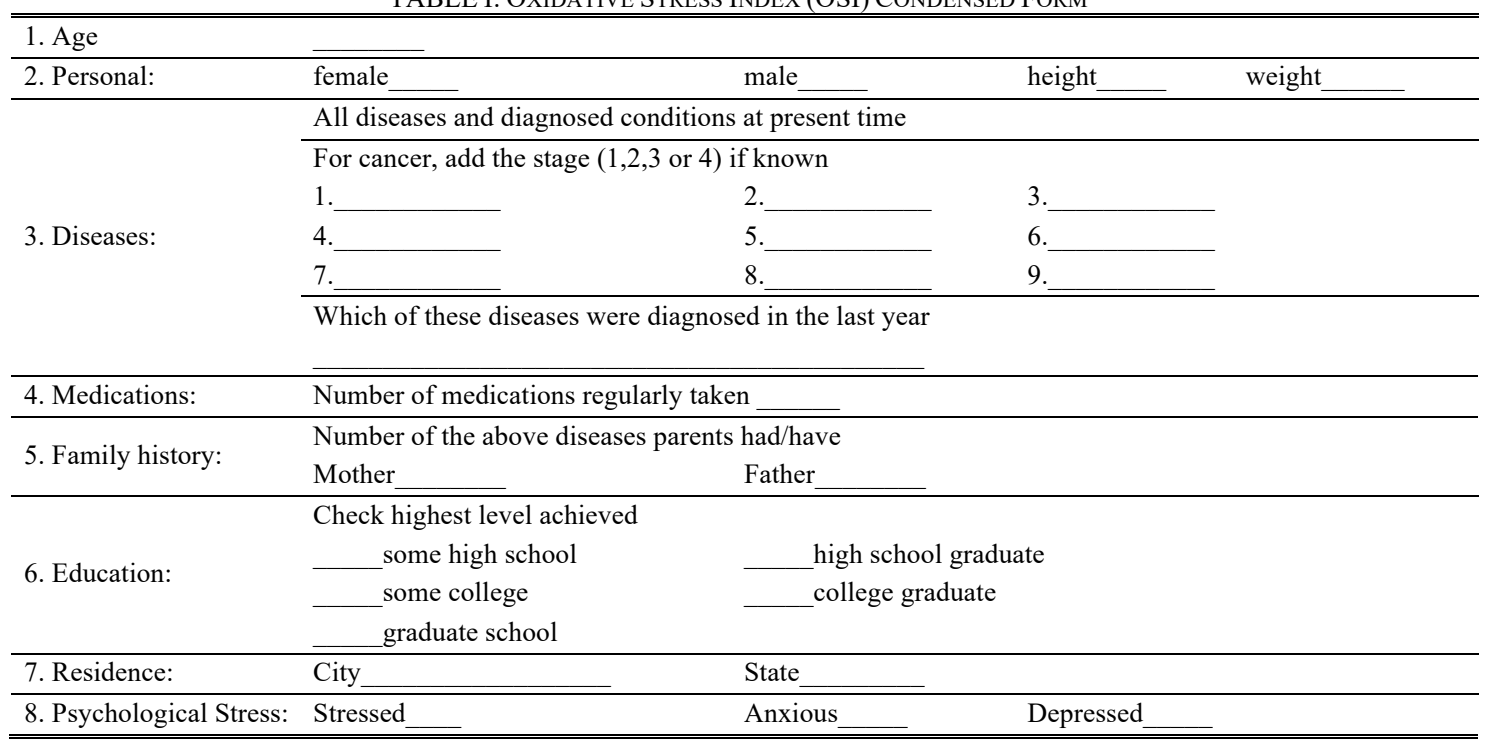

* Reprinted with permission from Zeliger HI. 2020. Oxidative Stress Index (OSI) condensed questionnaire. DOI:

http://dx.doi.org/10.24018/ejmed.2020.2.1.163.

The eight items in the condensed form are:

1. Age

2. Gender, height and Weight

3. Prevalent Disease

4. Medications Taken

5. Genetics

6. Socioeconomic Status - Education Level

7. Residence

8. Chronic Psychological Stress

As discussed below, these include all major sources of OS elevation and produce OSI values that are within 90 percent of those obtained using the detailed OSI questionnaire.

The OSI condensed form is based upon health impacts of the various parameters as reported in the literature. The scoring assignments for the individual parameters in the condensed form are based upon experience with the detailed OSI form. The point values assigned to each parameter are based upon those obtained via the detailed OSI questionnaire (Zeliger, 2017).

\section{1) Age}

Aging is accompanied by an increase in OS, generally starting around age 40 and continuing throughout life (Epel, et al., 2004; Hou, et al., 2015). In the condensed OSI one point is scored for each decade. For one aged 40-49, one OSI point is added to his or her total, 2 points are added for one aged 50-59, etc. (Zeliger, 2017).

\section{2) Gender, Height and Weight}

Being overweight is associated with elevated OS and the onset of numerous diseases. These include, but are not limited to; hypertension, dyslipidemia, type 2 diabetes, coronary heart disease, stroke, gallbladder disease, osteoarthritis, sleep apnea, breathing difficulties, anxiety and depression as well as endometrial, breast, colon, kidney, liver and gallbladder cancers (CDC, 2015). Gender, height and weight information enables one to determine if the person is of normal weight, is heavy or obese (Willett, 2001). The condensed OSI categorizes weight into three categories; normal, overweight and obese. Normal weight is assigned a value of zero. Heavy individuals are assigned a score of 1 and those who are obese are assigned a score of 2 .

\section{3) Chronic Prevalent Diseases}

The number of prevalent diseases at the time of OSI measurement is a critical indicator of OS. It is well established that all disease increases OS and hence the OSI (Zeliger, 2016; Zeliger 2017). All diseases have symptoms associated with them. These are individually addressed in the detailed OSI. The OSI condensed form does not probe individual symptoms. Rather, it has been found that assigning five points for each prevalent disease adequately 
incorporates elevations in OS associated with the prevalent diseases. Respondents are asked to list all diseases with which they have. Multiplying the number of prevalent diseases by five yields the disease number in the condensed OSI.

\section{4) Medications}

Essentially all pharmaceutical medications raise OS (Zeliger, 2017) and all have adverse drug reactions associated with them (Zeliger, 2019b). The detailed OSI form addresses these individually. In the condensed form, one OSI point is assigned for each medication taken.

\section{5) Genetics}

Genetics is well known to be a factor in most noncommunicable diseases with both genetic traits and epigenetic effects raising OS (Cencioni, et al., 2013; Dato, et al., 2013; Jiang, et al., 2013; Guillaumet-Adkins, et al., 2017). Though all non-communicable diseases are more prevalent in those whose ancestors have suffered from those diseases, parental disease is most closely associated with the likelihood of disease onset in an individual (Awdeh, et al., 2006). In the condensed OSI form, respondents are asked to check which of their prevalent diseases were also prevalent in their parents and each genetic link is assigned a value of one OSI point.

\section{6) Socioeconomic Status - Education Level}

Socioeconomic status (SES) is well established as an indicator of detrimental lifestyle choices that raise OS (Mielck, et al., 2014). These lifestyle choices include diets high in fats, sugar, salt or processed foods, tobacco, alcohol and recreational drug abuse, radiation exposure and the need to reside or work in a toxic chemical environment (Zeliger, 2016). SES is also associated with an increased likelihood of having undiagnosed diseases (Bein, et al., 2012; Mielck, et al., 2014; Shaw, et al., 2016).

Educational achievement has been shown to be a valid indicator of SES, with lower SES individuals more likely to lead unhealthy, OS raising life styles and to have undiagnosed diseases (Yin, et al., 2017). In the condensed OSI form, 5 education levels are identified and point values assigned for each as follows:

$\begin{array}{ll}\text { Some high school } & 5 \\ \text { High school graduate } & 4 \\ \text { Some college } & 3 \\ \text { College graduate } & 2 \\ \text { Graduate degree } & 1\end{array}$

\section{7) Residence}

In the $21^{\text {st }}$ century, 90 percent of the world's people, regardless of SES, are exposed to air pollution (Combes and Franchineau, 2019; World Health Organization, 2018). All air pollutants are toxic, raise $\mathrm{OS}$ in a dose response relationship, impact the OSI and are responsible for the onset of numerous diseases including respiratory diseases, cardiovascular diseases, several cancers and Alzheimer's disease (Combes and Franchineau, 2019; Zhou, et al., 2019; Xia, et al., 2019; Kilian J and Kitazawa M, 2018).

Recently, a method to quantify air pollution impact on disease onset, termed the Air Quality Toxicity Index (AQTI) has been reported (Zeliger, 2020). This method, based upon the dose response relationship between toxic exposure and OS elevation, enables the classification of air quality for individual locations to be calculated and reported as cumulative annual values.

In America, the United States Environmental Protection Agency (EPA) measures air quality in multiple locations on a daily basis and reports the data on-line as the Air Quality Index (AQI) (EPA, 2019). Worldwide, the World Air Quality Project similarly reports air quality data for hundreds of cities. (World Air Quality Index Project, 2019). On an annual basis, these indices identify the number of days in which the air quality in a given locale is classified as either good, moderate, unhealthy for sensitive groups, unhealthy, very unhealthy or hazardous. Hazard numbers ranging from $1-6$ have been assigned to the six air quality classifications, as follows (Zeliger, 2020a).

$\begin{array}{lc}\text { Air Quality Classification } & \text { Hazard Number } \\ \text { Good } & 1 \\ \text { Moderate } & 2 \\ \text { Unhealthy for Sensitive Groups } & 3 \\ \text { Unhealthy } & 4 \\ \text { Very Unhealthy } & 5 \\ \text { Hazardous } & 6\end{array}$

The air pollution impact on OSI is obtained by multiplying the number of days per year for each of the six EPA classifications at the residence of the responder by the hazard number for that classification and adding the these up to yield the AQTI total as shown in Table II. As also shown in Table II are the OSI air pollution severities (OSI - AP). These are assigned number values from $0-5$, and entered into the OSI condensed form.

TABLE II: ANNUAL AIR QUALITY CLASSIFICATION AS SHOWN BY AQTI

\begin{tabular}{ll}
\hline \hline AIR QUALITY CLASSIFICATION & AQTI \\
\hline Good & $365-450$ \\
\hline Moderate & $451-600$ \\
\hline Unhealthy for Sensitive Groups & $601-750$ \\
\hline Unhealthy & $751-900$ \\
\hline Very Unhealthy & $901-1050$ \\
\hline Hazardous & 1051 or higher \\
\hline \hline
\end{tabular}

\section{8) Chronic Psychological Stress}

Psychological stress, anxiety and depression are associated with most diseases and are more pronounced as illnesses progress (Sahle, et al., 2020). Each of these three conditions raises OS via the release of hormones that elevate OS. Chronic activation of this stress response system results in disease and triggers numerous health problems (Mayo Clinic, 2019).

Respondents are asked to check if they often feel stressed, anxious or depressed. One point is assigned for each positive response.

The relevance of the OSI to predicting disease onset probability as well as for other applications uses has been previously established (Zeliger, 2017, 2019, 2019a, 2019b, 2019 c). When the OSI condensed form values are used as presented here an excellent correlation between standard form and condensed form OSI values occurs, with condensed form values showing less than a $10 \%$ variation from standard form values.

To sum up, the point values assigned to each of the 
parameters contained in the OSI condensed questionnaire are as follows:

Age: 1 for each decade of age starting at age 40.

Personal: 0,1, or 2, depending upon weight status

Diseases: 3 per prevalent chronic disease

Medications: 1 per prescribed or over-the-counter medication regularly taken

Genetics: 1 per each item checked

Education:1 to 5, depending upon highest education level achieved

Residence: 0 to 5, depending upon air pollution level at residence

Stress:0 to 3 depending upon chronic psychological status

\section{OSI VALUES AND DiseASE LIKELIHOOD}

The following scale of OSI values has been associated with the likelihood of disease onset. It is suggested here that the same scale can be used to predict COVID-19 severity.

$\begin{array}{ll}0-15 & \text { Indicative of good health } \\ 16-30 & \text { Disease predicted } \\ 31-45 & \text { Disease probable } \\ 45 \text { or greater } & \text { Disease imminent }\end{array}$

\section{COVID-19}

The relevance of OS and the OSI in predicting COVID19 severity is based on the following:

1. COVID-19 disease has been shown to be of greater severity and resulting in increased numbers of deaths in people aged 65 years and older and those with preexisting conditions that include hypertension, cardiovascular disease, diabetes, air pollution exposure and smoking history (Emami, et al., 2020; Zhang, et al., 2020; Zhou, et al., 2020; Wu, et al., 2020).

2. Pre-existing diseases and smoking all elevate OS and the OSI (Zeliger, 2016; Zeliger, 2019).

3. Viral diseases are more prevalent and more severe in those with lifestyles that include OS raising practices such as smoking, vaping and regular alcohol consumption (Khomich, et al., 2018; Gualano, et al., 2008).

4. Obesity, which also raises OS, is a risk factor for increased viral disease severity (Honce \& SchultzCherry, 2019).

5. Lifestyles that reduce OS, such as following the Mediterranean diet, have been proposed to protect against COVID-19 severity (Hopkins, 2020).

6. In the United States, young residents of the deep south and mid-south states have been found to be at much higher risk of death from COVID-19 than their similarly aged cohorts in other states. This effect has been attributed to a higher prevalence of heart disease, lung disease, diabetes, obesity and tobacco smoking (Keiser Family Foundation, 2020).

It has been previously shown that lowering oxidative stress via lifestyle changes and appropriate treating of preexisting conditions can prevent the onset of numerous chronic diseases and reduce the severity of pre-existing ones
(Zeliger, 2016). Use of the OSI has also been shown to be helpful in exploring which of the known contributors to the onset of Alzheimer's disease are more prevalent (Zeliger, 2019a). It is proposed here that a similar approach can potentially be used to ascertain if particular pre-existing conditions are more prevalent in the severity of COVID-19 after its onset or if, indeed, severity is a function of total oxidative stress, as it the case with other viral infections, such as the common cold (Khomich, et al., 2018). OSI screening of current COVID-19 patients can also potentially be used retroactively to associate OSI ranges with degree of disease severity.

The condensed form of the OSI readily lends itself to an electronic app that can be accessed from computers or cell phones and instantly produces a numerical OSI value. Results can be applied on both micro and macro scales.

On a micro scale, using the condensed form of the OSI, individuals can pre-screen themselves to determine if their pre-existing conditions suggest the potential for severe disease and, if so, seek medical screening for the presence of COVID-19, prior to symptom onset. OSI results can be used to indicate life-style choices that can lower OS and thus potentially lower the severity of COVID-19 disease.

On a macro scale, results from OSI screening can potentially be used to predict the number of COVID-19 cases that can be anticipated in a given locale and thus help health care professionals predict the amount of care that will be required and allocate resources appropriately. The knowledge learned from application of the OSI to COVID19 can also be applied to future epidemics and pandemics.

Though the hypothesis proposed here is yet unproven, there is no risk in applying the OSI to the prediction of COVID-19 severity, for the procedure is not invasive, and only recommends lifestyle changes that cannot harm. Indeed, lowering OS has been shown to prevent the onset of numerous chronic diseases and reduce severity of preexisting diseases.

\section{CONCLUSIONS}

It is proposed here that the non-invasive Oxidative Stress Index can be used to determine the association between oxidative stress level and severity of COVID-19 disease. Once determined, the OSI can potentially predict a particular individual's risk for severity of the disease as well as lifestyle changes that might reduce disease severity. The OSI could also be used to predict the amount of preparation necessary for treatment of COVID-19 and demonstrate vulnerability to disease onset. Potentially, the OSI could potentially be applied future epidemics and pandemics. Lowering OS via lifestyle changes such as eating a healthy diet and ceasing smoking cannot harm but could very well improve a person's overall health.

\section{REFERENCES}

Akaike T. 2001. Role of free radicals in viral pathogenesis and mutation. Rev Med Virol; 11(2):87-101.

Awdeh ZL, Yunis EJ, Auden MJ, Fici C, Pugliese A, Larsen CE, Alper CA. 2006. A genetic explanation for the rising incidence of type 1 diabetes, a polygenic disease. J Autoimmun; 27(3):174-181.

Bein T, Hackner K, Zou T, Schultes S, Bosch T, Schlitt HJ, et al. 2012 Socioeconomic status, severity of disease and level of family 
members' care in adult surgical intensive care patients: the prospective ECSSTASI study. Intensive Care Med; 38:612-619.

CDC. 2015. Centers for Disease Control and Prevention. Healthy weight. The health effects of overweight and obesity https://cdc.gov/healthyweight/effects/index.html_Accessed January 25,2020

Cencioni C, Spalotta F, Martelli F, Valente S, Mai A, Zeiher AM, Gaetano C. Oxidative stress and epigenetic regulation in aging and are-related diseases. Int J Mol Sci; 14:17643-17663.

Combes A, Franchineau G. 2019. Fine particle environmental pollution and cardiovascular diseases. Metabolism, 2019. doi: 00.16/j.metabol.2019.07.008Accessed January 27, 2020.

Dato S, Crocco P, D’Aquila P, de Rango F, Belllizi D, Rose G, Passarino G. 2013. Exploring the role of genetic variability and lifestyle in oxidative stress response for healthy aging and longevity, Int $\mathrm{J}$ Mol Sci; $14: 16443-16472$

Emami A, Javanmardi F, Pirbonyeh N, Akbari A. 2020. Arch Acad Emerg Med; 8(1):e35. eCollection 2020. Accessed March 31, 2020.

EPA. 2019. Air quality index (AQI) https:/airnow.gov/index.cfm?action=aquibasics.aqi Accessed January 2, 2020.

Epel ES, Blackburn EH, Dhabhar FS, Adler NE, Morrow JD, Cawthorn RM. 2014. Accelerated telomere shortening in response to life stress. Proc Nat Acad Sci USA 101(49):17312-17315.

Gualano RC, Hansen MJ, Vlahos R, Jones JE, Park-Jones RA, Deliyannis $\mathrm{G}$, et al. 2008. Cigarette smoke worsens lung inflammation and impairs resolution of influenza infection in mice. Resp Res; 9:53 doi: 10.1186/1465-9921-9-53. Accesssed February 28, 2020.

Guillaumet-Adkins A, Yanez Y, Preis MD, Palancia-Ballester C, Sandoval J. 2017.Ox Med Cell Longevity article No. ID9175806. Doi: 10.1155/2017/9175806. Accessed January 23, 2020.

Honce R, Schultz-Cherry S. 2019. Impact of obesity on Influenza A virus parthenogenesis, immune response and evolution. Front Immunol 10;10:1071. Doi: 10.3389/fimmu.2019.01071. eCollection 2019 Accessed April 4, 2020

Honjo K, von Reeku R, Verhoeff NP. 2009. Alzheimer's disease and infection: do infectious agents contribute to progression of Alzheimer's disease? Alz Dement; 5(4):348-60.

Hopkins. 2020. Take your diet to the Mediterranean https://www.hopkinsmedicine.org/healthlibrary/conditions/adult/cardi ovascular diseases/atherosclerosis. Accessed April 4, 2020

Hou L, Joyce BT, Gao T, Liu L, Zheng Y, Penedo FJ, et al. 2015. Blood telomere length attrition and cancer development in the normative aging study cohort. EBioMedicine 132(6):591-596.

Hughes DA. 1999. Effects of dietary antioxidants on the immune function of middle-aged adults. Proc Nutr Soc; 58:79-84.: E393. Doi: 10.3390/v10080392. Accessed March 14, 2020.

Jiang T, Yu JT, Tian Y, Tan L. 2013. Epidemiology and etiology of Alzheimer's disease. From genetic to non-genetic factors. Curr Alzheimer Res; 9:852-867.

Khomich OQ, Kochetkov SN, Bartosch B, Ivanov AV. 2018. Redox biology of respiratory viral infections. Viruses; 10(8). Pii

Kilian J, Kitazawa M. 2018. The emerging risk of exposure to air pollution on cognitive decline and Alzheimer's disease - Evidence from epidemiological and animal studies. Biomed J 41(3):141-162.

Mayo Clinic. 2019. Healthy lifestyle stress management https://www.mayoclinic.org/heaalthy-lifestyles-management/indepth/stress/art-20046037. Accessed April 1, 2020.

Marais BJ, Lonroth K, Lawn SD, Migliori GB, Mwaba K, Glaziou P, et al. 2013. Tuberculosis comorbidity with communicable and noncommunicable diseases: integrating health services and control efforts. Lancet Infect Dis; 13(5):436-48.

Mielck A, Vogelman M, Reiner L. 2014. Health-related quality of life and socioeconomic status: inequalities among adults with a chronic disease. $\quad$ BioMed $\quad$ Central; $12: 58$ http://www.hqlo.com/content/12/1/58. Accessed January 21, 2020.

Pizzino G, Irrera N, Cucinotta M, Pallio G. Mannino F, Arcoraci V, et al. Oxidative stress: Harms and benefits for Human Health. 2017 Oxidative Health and Cellular Longenvity; 2017 Article ID 8416763. https://doi.org/10.1155/2017/8416763. Accessed April 1, 2020.

Spettstoesser WD, Schuff-Werner P. 2002 Oxidative stress in phagocytes the enemy within. Microsc Res Tech; 57(6):441-55.

Nielsen F, Mikkelsen BB, Niesen JB,Andesen HR, Grandjean P. 1997. Plasma malondialdehyde as biomarker for oxidative stress: reference onterval and efforts of life-style factors. Clin Chem; 43(7):1209-14.

Rajah T, Chow SC. 2015. Suppression of human T cell proliferation mediated by the Cathepsin B inhibitor z-FA-FMK is due to oxidative stress. PLoS One; 10(4):e123711. Accessed March 22, 2020.
Sahle BW, Chen W, Melaku YA, Akombi BJ, Rawal LB, Renzaho AMN. 2020. Association of psychosocial factors with risk of chronic diseases: A nationwide longitudinal study. Am J Prev Med; 58(2):e39-e50. Doi: 10.1016/j.amepre.2019.09.007 Accessed January $27,2020$.

Shaw KM, Theis KA, Self-Brown S, Roblin DW, Barker L. 2016. Chronic disease disparities by county economic status and metropolitan classification, behavioral risk factor surveillance system, 2013. Centers for Disease Control. Preventing chronic disease. https://www.cdc.giv/pcd/issues/2016/16_0088.htm. Accessed January $21,2020$.

Willett WC. 2001. Eat, drink and be happy. Free Press, New York.

World Air Quality Index Project. 2019. https://aqicn.com. Accessed January 27, 2020.

Wu X, Nethery RC, Sabath MB, Braun D, Dominici F. 2020. Exposure to air pollution and COVID-19 mortality in the United States. JAMA, In press.

Xia SY, Huang DS, Jia H, Zhao Y, Lin N, Mao MQ, et al. 2019 Relationship between atmospheric pollutants and risk of death caused by cardiovascular and respiratory diseases and malignant tumors in Shenyang, China from 2013to 2016: an ecological research. Chin Med J (Engl); 132(19):2269-2277.

Xu GJ, Kula T, Xu Q, Li MZ, Vernon SD, Ndung'n T, et al. 2015. Comprehensive serological profiling of human populations using a synthetic human virome. Science; 348:aaa0698

Yin H, Wu Q, Cui Y, Hao Y, Liu C, Li Y, et al. 2017. Socioeconomic status and prevalence of chronic non-communicable diseases in Chinese women: a structural equation modelling approach. BMJ Open 2017;7:e014402. doi: 10.1136/bmjopen-2016-014402. Accessed January 22, 2020.

Zaki MH, Akuta T, Akaike T. 2005. Nitric oxide-induced nitrative stress involved in microbial parthenogenesis. J Pharmacol Sci; 98:117-29.

Zeliger HI, Pan Y, Rea WJ. 2012. Predicting co-morbidities in chemically sensitive individuals from exhaled breath analysis. Interdiscip Toxicol; 5(3):123-26.

Zeliger HI. 2014. Co-morbidities of environmental diseases: a common cause. Interdisip Toxicol 7(3):117-122.

Zeliger HI. 2016. Predicting disease onset in clinically healthy people. Interdisip Toxicol; $9(2): 39-54$

Zeliger HI. 2017. Oxidative Stress Index predicts disease onset. J Med Res Pract; 6(3):86-92. doi.org/10.20936/jmrp/17/03/003. Accessed April $1,2020$.

Zeliger HI. 2019. EC Pharmacology and Toxicology; 7.9 (2019):1022-36.

Zeliger HI. 2019a. Predicting Alzheimer's disease onset. Euro J Med Health Sci; 1(1). DOI: http://dx.doi.org/10.24018/ejmed.2019.1.1.16. Accessed April 1, 2020.

Zeliger HI. 2019b Predicting and reducing adverse drug reactions. Euro J Med Health Sci; 1(4). http://dx.doi.org/10.24018/ejmed.2019.1.4.91 Accessed January 22, 2020 .

Zeliger HI. 2020. Oxidative Stress Index (OSI) condensed questionnaire. DOI: http://dx.doi.org/10.24018/ejmed.2020.2.1.163. Accessed March 30,2020

Zeliger HI. 2020a. Air quality toxicity index (AQTI): Quantifying air pollution impact on disease onset. Eur J Med Health Sci; 2(1). DOI: http:dx.doi.org/10.24018/ejmed.2020.2.1.143. Accessed January 28, 2020.

Zhang JJ, Dong X, Cao YY, Yuan YD, Yang YB, Yan YQ, et al. 2020. Clinical characteristics of 140 patients infected with SARS-CoV-2 in Wuhan, China. Allergy; doi: 10,1111/all.14238. Accessed April 4, 2020.

Zhou F, Yu T, Du R, Guohui F, Liu Y, Liu Z, et al. 2020. Clinical course and risk factors for mortality of adult inpatients with COVID-19 in Wuhan, China: a retrospective cohort study. Lancet. https://doi.org/1016/50140-6736(20)30566-3. Accessed April 2, 2020.

Zhou H, Wang T, Zhou F, Liu Y, Zhao W, Wang X, et al. 2019. Ambient air pollution and daily hospital admissions for respiratory disease in children in Guiyang, China. Front Pediatr. 2019. Doi: 10.3389/fped.2019.00400. Accessed January 30, 2020. 